\title{
On the Approximation of Generalized Lipschitz Function by Euler Means of Conjugate Series of Fourier Series
}

\author{
Jitendra Kumar Kushwaha \\ Department of Pure \& Applied Mathematics, Guru Ghasidas University, Koni, Bilaspur 495009, India \\ Correspondence should be addressed to Jitendra Kumar Kushwaha; k.jitendrakumar@yahoo.com \\ Received 8 August 2013; Accepted 26 September 2013 \\ Academic Editors: K. A. Gepreel and Q. Xie
}

Copyright ( 2013 Jitendra Kumar Kushwaha. This is an open access article distributed under the Creative Commons Attribution License, which permits unrestricted use, distribution, and reproduction in any medium, provided the original work is properly cited.

\begin{abstract}
Approximation theory is a very important field which has various applications in pure and applied mathematics. The present study deals with a new theorem on the approximation of functions of Lipschitz class by using Euler's mean of conjugate series of Fourier series. In this paper, the degree of approximation by using Euler's means of conjugate of functions belonging to $\operatorname{Lip}(\xi(t)$, $p)$ class has been obtained. $\operatorname{Lip} \alpha$ and $\operatorname{Lip}(\alpha, p)$ classes are the particular cases of $\operatorname{Lip}(\xi(t), p)$ class. The main result of this paper generalizes some well-known results in this direction.
\end{abstract}

\section{Introduction and Definitions}

Let $f$ be periodic with period $2 \pi$ and integrable in the sense of Lebesgue. The Fourier series associated with $f$ at the point $x$ is given by

$$
f(x) \sim \frac{1}{2} a_{0}+\sum_{n=1}^{\infty}\left(a_{n} \cos n x+b_{n} \sin n x\right)=\sum_{n=0}^{\infty} A_{n}(x)
$$

with partial sums $s_{n}(f ; x)$. The conjugate series of (1) is given by

$$
B_{n}(x)=\sum_{n=1}^{\infty}\left(a_{n} \sin n x-b_{n} \cos n x\right)
$$

with partial sums $\widetilde{s}_{n}(f ; x)$. Throughout this paper, we call (2) as conjugate series of Fourier series of function $f$. If $f$ is Lebesgue integrable, then

$$
\begin{aligned}
\tilde{f}(x) & =-\frac{1}{2 \pi} \int_{0}^{\pi} \psi(t) \cot \left(\frac{t}{2}\right) d t \\
& =-\frac{1}{2 \pi} \lim _{\varepsilon \rightarrow 0} \int_{\varepsilon}^{\pi} \psi(t) \cot \left(\frac{t}{2}\right) d
\end{aligned}
$$

exists for almost all $x$ (Hardy [1], page 131). $\widetilde{f}(x)$ is called the conjugate function of $f(x)$.
A function $f \in \operatorname{Lip} \alpha$ if

$$
|f(x+t)-f(x)|=O\left(|t|^{\alpha}\right) \quad \text { for } 0<\alpha \leq 1 .
$$

$f \in \operatorname{Lip}(\alpha, p), p>1$ consider that if

$$
\begin{array}{r}
\left\{\int_{0}^{2 \pi}|f(x+t)-f(x)|^{p} d x\right\}^{1 / p}=O\left(|t|^{\alpha}\right), \\
0<\alpha \leq 1, p \geq 1
\end{array}
$$

(Definition 5.38 of Chandra [2]).

Given a positive increasing function $\xi(t), f \in$ $\operatorname{Lip}(\xi(t), p)$,

$$
\begin{array}{r}
\left(\int_{0}^{2 \pi}|(f(x+t)-f(x))|^{p} d x\right)^{1 / p} \leq M\left(\xi(t) t^{-1 / p}\right), \\
p>1,
\end{array}
$$

where $M$ is a positive number independent of $x$ and $t$.

In case $\xi(t)=t^{\alpha}$, then $\operatorname{Lip}(\xi(t), p)$ coincides with $\operatorname{Lip}(\alpha, p)$. If $p \rightarrow \infty$ in $\operatorname{Lip}(\alpha, p)$, then it coincides with Lip $\alpha$.

$L_{\infty}$-norm of a function $f: R \rightarrow R$ is defined by

$$
\|f\|_{\infty}=\sup \{|f(x): x \in R|\},
$$


$L_{p}$-norm is defined by

$$
\|f\|_{p}=\left(\int_{0}^{2 \pi}|f(x)|^{p} d x\right)^{1 / p}, \quad p \geq 1 .
$$

The degree of approximation of a function $f: R \rightarrow R$ by a trigonometric polynomial $t_{n}$ of order $n$ under sup norm \|\|$_{\infty}$ is defined by ([1], page 114-115])

$$
\left\|t_{n}-f\right\|_{\infty}=\sup \left\{\left|t_{n}(x)-f(x)\right|: x \in R\right\},
$$

and $E_{n}(f)$ of a function $f \in L_{p}$ is given by

$$
E_{n}(f)=\min \left\|t_{n}-f\right\|_{p} .
$$

Let $\left\{S_{n}\right\}$ be the sequence of partial sums of the given series $\sum_{n=0}^{\infty} u_{n}$. Then, for $q>0$, the Euler $(E, q)$ means of $\left\{S_{n}\right\}$ are defined to be

$$
W_{n}=(1+q)^{-n} \sum_{k=0}^{n}\left(\begin{array}{l}
n \\
k
\end{array}\right) q^{n-k} S_{k}
$$

The series is said to be Euler $(E, q)$ summable to $S$ provided that the sequence $\left\{W_{n}\right\}$ converges to $S$ as $n \rightarrow+\infty$.

We write

$$
\begin{gathered}
\psi(t)=f(x+t)-f(x-t), \\
\widetilde{\sigma}_{n}(f ; x)=(1+q)^{-n} \sum_{k=0}^{n}\left(\begin{array}{l}
n \\
k
\end{array}\right) q^{n-k} \widetilde{S}_{k}, \\
\widetilde{S}(t)=\sum_{k=0}^{n}\left(\begin{array}{l}
n \\
k
\end{array}\right) q^{n-k} \cos \left(k+\frac{1}{2}\right) t, \\
R(t)=\sin \left\{\frac{t}{2}+n \tan ^{-1}\left(\frac{\sin t}{q+\cos t}\right)\right\} .
\end{gathered}
$$

\section{Main Theorem}

Hardy [1] established a theorem on $(C, \alpha),(\alpha>0)$ summability of the series. Harmonic summability is weaker than $(C, \alpha)$ summability. Iyengar [3] proved a theorem on harmonic summability of a Fourier series. The result of Iyengar [3] has been generalized by several researchers like Siddiqi [4], Pati [5], Lal and Kushwaha [6], and Rajagopal [7], for Nörlund means.

Alexits [8] proved the following theorem concerning the degree of approximation of a function $f \in \operatorname{Lip} \alpha$ by the $(C, \delta)$ means of its Fourier series.

Theorem A. If a periodic function $f \in \operatorname{Lip} \alpha, 0<\alpha \leq 1$, then the degree of approximation of the $(C, \delta)$ means of its Fourier series for $0<\alpha<\delta \leq 1$ is given by

$$
\max _{0 \leq x \leq 2 \pi}\left|f(x)-\sigma_{n}^{\delta}(x)\right|=O\left(\frac{1}{n^{\alpha}}\right)
$$

and for $0<\alpha \leq \delta \leq 1$ is given by

$$
\max _{0 \leq x \leq 2 \pi}\left|f(x)-\sigma_{n}^{\delta}(x)\right|=O\left(\frac{\log n}{n^{\alpha}}\right),
$$

where $\sigma_{n}^{\delta}(x)$ are the $(C, \delta)$ means of the partial sums of (2).
Later on, Hölland et al. [9] extended Theorem A to functions belonging to $C *[0,2 \pi]$, the class of $2 \pi$-periodic continuous functions on $[0,2 \pi]$, using Nörlund means of Fourier series. Their theorem is as follows.

Theorem B. If $w(t)$ is the modulus of continuity of $f \in C *$ $[0,2 \pi]$, then the degree of approximation of $f$ by the Nörlund means of the Fourier series for $f$ is given by

$$
E_{n}=\max _{0 \leq t \leq 2 \pi}\left|f(t)-T_{n}(t)\right|=O\left(\frac{1}{p_{n}} \sum_{k=1}^{n} \frac{p_{k} w(1 / k)}{k}\right),
$$

where $T_{n}$ are the $\left(N, p_{n}\right)$ means of Fourier series off.

Hölland et al. [9] have shown that Theorem $\mathrm{B}$ reduces to Theorem $\mathrm{A}$ if we deal with Cesàro means of order $\delta$ and consider a function $f \in \operatorname{Lip} \alpha, 0<\alpha \leq 1$. Working in same direction we prove the following theorem.

Theorem 1. If $f: R \rightarrow R$ is a $2 \pi$ periodic, Lebesgue integrable and belonging to $\operatorname{Lip}(\xi(t), p)$ for $>1$ and if

$$
\begin{aligned}
& \left\{\int_{0}^{1 / \sqrt{n}}\left(\frac{t|\xi(t)|}{t^{1 / p}}\right)^{p} d t\right\}^{1 / p}=O\left(\xi\left(\frac{1}{\sqrt{n}}\right)\right), \\
& \left\{\int_{1 / \sqrt{n}}^{\pi}\left(\frac{|\xi(t)|}{t^{1 / p+2}}\right)^{p} d t\right\}^{1 / p}=O\left(\xi\left(\frac{1}{\sqrt{n}}\right) n\right)
\end{aligned}
$$

conditions (16) and (17) hold uniformly in $x$, then degree of approximation of $\tilde{f}(x)$, conjugate of $f \in \operatorname{Lip}\{\xi(t), p\}$, by Euler $(E, q)$ mean

$$
\widetilde{\sigma}_{n}(f ; x)=(1+q)^{-n} \sum_{k=0}^{n}\left(\begin{array}{l}
n \\
k
\end{array}\right) q^{n-k} \widetilde{S}_{k},
$$

of the conjugate series (2) is given by

$$
\left\|\widetilde{\sigma}_{n}(f ; x)-\tilde{f}(x)\right\|_{p}=O\left(\xi\left(\frac{1}{\sqrt{n}}\right)(n)^{1 / 2 p}\right) .
$$
lemma.

In order to prove our theorem, we need the following

Lemma 2. If $0<t \leq \pi$, then

$$
(1+q)^{-n}\left(1+q^{2}+2 q \cos t\right)^{n / 2} \leq e^{-2 q t^{2} n /\{\pi(1+q)\}^{2}} .
$$

Proof. We have

$$
\begin{gathered}
(1+q)^{-2}\left(1+q^{2}+2 q \cos t\right) \\
=1-\frac{4 q \sin ^{2}(t / 2)}{(1+q)^{2}} \\
\leq 1-\frac{4 q t^{2}}{\pi^{2}(1+q)^{2}} \\
\leq e^{-4 q t^{2} /\{\pi(1+q)\}^{2}},
\end{gathered}
$$


since $e^{x}(1-x)<1$ when $0<x<1$. Therefore,

$$
(1+q)^{-n}\left(1+q^{2}+2 q \cos t\right)^{n / 2} \leq e^{-2 q t^{2} n /\{\pi(1+q)\}^{2}} .
$$

Proof of Theorem 1. The $k$ th partial sum of the conjugate series of the Fourier series (2) is given by

$$
\begin{aligned}
& \widetilde{S}_{k}(f ; x)=-\frac{1}{2 \pi} \int_{0}^{\pi} \cot \left(\frac{t}{2}\right) \psi(t) d t \\
&+\frac{1}{2 \pi} \int_{0}^{\pi} \frac{\cos (k+1 / 2) t}{\sin (t / 2)} \psi(t) d t \\
& \widetilde{S}_{k}(f ; x)-\left(-\frac{1}{2 \pi} \int_{0}^{\pi} \cot \left(\frac{t}{2}\right) \psi(t) d t\right) \\
&=\frac{1}{2 \pi} \int_{0}^{\pi} \frac{\cos (k+1 / 2) t}{\sin (t / 2)} \psi(t) d t .
\end{aligned}
$$

Taking Euler $(E, q)$ means, we get

$$
\begin{aligned}
{[(1} & \left.+q)^{-n} \sum_{k=0}^{n}\left(\begin{array}{l}
n \\
k
\end{array}\right) q^{n-k}\left\{\widetilde{S}_{k}(f ; x)-\widetilde{f}(x)\right\}\right] d t \\
= & \frac{1}{\pi(1+q)^{n}} \int_{0}^{\pi} \frac{\psi(t)}{\sin (t / 2)} \\
& \times\left\{\sum_{k=0}^{n}\left(\begin{array}{l}
n \\
k
\end{array}\right) q^{n-k} \cos \left(k+\frac{1}{2}\right) t\right\} \\
\widetilde{\sigma}_{n}(f ; x)-\widetilde{f}(x) & \frac{1}{\pi(1+q)^{n}} \int_{0}^{\pi} \frac{\psi(t)}{\sin (t / 2)} \widetilde{S}(t) d t \\
= & \frac{1}{\pi(1+q)^{n}}\left[\int_{0}^{1 / \sqrt{n}}+\int_{1 / \sqrt{n}}^{\pi}\right] \frac{\psi(t)}{\sin (t / 2)} \widetilde{S}(t) d t \\
= & \widetilde{K}_{1}(x)+\widetilde{K}_{2}(x),
\end{aligned}
$$$$
\frac{\widetilde{S}(t)}{(1+q)^{n}} \leq \frac{1}{(1+q)^{n}}
$$$$
\times\left|\sum_{k=0}^{n}\left(\begin{array}{l}
n \\
k
\end{array}\right) q^{n-k} e^{i(k+1 / 2) t}\right|
$$$$
=\frac{\left|q+e^{i t}\right|^{n}}{(1+q)^{n}}=\frac{\left(1+q^{2}+2 q \cos t\right)^{n / 2}}{(1+q)^{n}}
$$$$
\leq e^{-2 q t^{2} n /\{\pi(1+q)\}^{2}},
$$

using Lemma 2

Clearly,

$$
\begin{aligned}
|\psi(x+t)-\psi(x)| \leq & |f(u+x+t)-f(u+x)| \\
& +|f(u+x)-f(u-x-t)| .
\end{aligned}
$$

Hence, by Minkowski’s inequality,

$$
\begin{aligned}
& \left\{\int_{0}^{2 \pi}|(\psi(x+t)-\psi(x))|^{p} d x\right\}^{1 / p} \\
& \leq\left\{\int_{0}^{2 \pi}|(f(u+x+t)-f(u+x))|^{p} d x\right\}^{1 / p} \\
& \quad+\left\{\int_{0}^{2 \pi}|f(u+x)-(f(u-x-t))|^{p} d x\right\}^{1 / p} \\
& =O(\xi(t)) .
\end{aligned}
$$

Then, $f \in \operatorname{Lip}(\xi(t), p) \Rightarrow \psi \in \operatorname{Lip}(\xi(t), p)$.

Using Hölder's inequality, $\psi(t) \in \operatorname{Lip}(\xi(t), p)$, condition (16), $\sin t \geq(2 t / \pi)$, lemma, and second mean value theorem for integrals, we have

$$
\begin{aligned}
\left|\widetilde{K}_{1}(x)\right|= & O\left\{\int_{0}^{1 / \sqrt{n}}(|\psi(t)|)^{p} d t\right\}^{1 / p} \\
& \times\left\{\int_{0}^{1 / \sqrt{n}}\left(\frac{(1+q)^{-n} \widetilde{S}(t)}{\sin (t / 2)}\right)^{p^{\prime}} d t\right\}^{1 / p^{\prime}},
\end{aligned}
$$

where

$$
\begin{aligned}
p^{\prime}= & \frac{p}{p-1}\left|\widetilde{K}_{1}(x)\right| \\
= & O\left\{\int_{0}^{1 / \sqrt{n}}\left(\left|\frac{\xi(t)}{t^{1 / p}}\right|\right)^{p} d t\right\}^{1 / p} \\
& \times\left\{\int_{0}^{1 / \sqrt{n}}\left(\frac{e^{-2 q t^{2} n /\{\pi(1+q)\}^{2}}}{|\sin (t / 2)|}\right)^{p^{\prime}} d t\right\}^{1 / p^{\prime}} \\
= & O\left(\xi\left(\frac{1}{\sqrt{n}}\right)\right)\left\{\int_{0}^{1 / \sqrt{n}} t^{-p^{\prime}} d t\right\}^{1 / p^{\prime}} \\
= & O\left(\xi\left(\frac{1}{\sqrt{n}}\right)(n)^{1 / 2 p}\right) .
\end{aligned}
$$

Now,

$$
\begin{aligned}
&\left|\widetilde{K}_{2}(x)\right|=O {\left[\begin{array}{c}
\int_{1 / \sqrt{n} \sin (t / 2)}^{\pi} \frac{|\psi(t)|}{\sin }(1+q)^{-n} \\
\left(1+q^{2}+2 q \cos t\right)^{n / 2}|R(t)| d t
\end{array}\right] } \\
&=O\left\{\int_{1 / \sqrt{n}}^{\pi} \frac{|\psi(t)|}{\sin (t / 2)}(1+q)^{-n}\right. \\
&\left.\times\left(1+q^{2}+2 q \cos t\right)^{n / 2} d t\right\}
\end{aligned}
$$




$$
\begin{gathered}
=O\left\{\int_{1 / \sqrt{n}}^{\pi} \frac{|\psi(t)|}{\sin (t / 2)} e^{-2 q t^{2} n /\{\pi(1+q)\}^{2}} d t\right\} \\
=O\left[\frac{1}{n} \int_{1 / \sqrt{n}}^{\pi} \frac{\psi(t)}{\sin (t / 2)}\right. \\
\left.\times\left\{\frac{\partial}{\partial t}\left(e^{-2 q t^{2} n /\{\pi(1+q)\}^{2}}\right)\right\} d t\right] .
\end{gathered}
$$

Using Hölder's inequality, $\psi(t) \in \operatorname{Lip}(\xi(t), p)$, and condition (17), we have

$$
\begin{aligned}
\left|\widetilde{K}_{2}(x)\right|= & O\left[\frac{1}{n} \int_{1 / \sqrt{n}}^{\pi}\left(\frac{\xi(t)}{t^{1 / p+2}}\right)^{p} d t\right]^{1 / p} \\
& \times\left[\int_{1 / \sqrt{n}}^{\pi}\left\{\frac{\partial}{\partial t}\left(e^{-2 q t^{2} n /\{\pi(1+q)\}^{2}}\right)\right\}^{p^{\prime}} d t\right]^{1 / p^{\prime}} \\
= & O\left(\xi\left(\frac{1}{\sqrt{n}}\right)(n)^{1 / 2 p}\right) .
\end{aligned}
$$

Combining (24) with (31), we have

$$
\left\|\widetilde{\sigma}_{n}(f ; x)-\tilde{f}(x)\right\|_{p}=O\left(\xi\left(\frac{1}{\sqrt{n}}\right)(n)^{1 / 2 p}\right),
$$

which completes the proof of the theorem.

\section{Corollaries}

The following corollaries may be derived from our theorem.

Corollary 3. If $\xi(t)=t^{\alpha}$, then the degree of approximation of a function $\tilde{f}(x)$, conjugate of $f \in \operatorname{Lip}(\alpha, p), 1 / p<\alpha<1$, by Euler's means $(E, q)$ of the conjugate series of the Fourier series (2) is given by

$$
\left\|\widetilde{\sigma}_{n}(f ; x)-\tilde{f}(x)\right\|_{p}=O\left(\frac{1}{n^{(\alpha p-1) / 2 p}}\right) .
$$

Corollary 4. If $p \rightarrow \infty$ in Corollary 3 , then, for $0<\alpha<1$,

$$
\left\|\widetilde{\sigma}_{n}(f ; x)-\tilde{f}(x)\right\|_{\infty}=O\left(\frac{1}{n^{\alpha / 2}}\right) .
$$

\section{References}

[1] G. H. Hardy, "On the summability of Fourier series," Proceedings of the London Mathematical Society, vol. 12, pp. 365-372, 1913.

[2] P. Chandra, "On the degree of approximation of functions belonging to the lipschitz class," Nanta Mathematica, vol. 8, no. 1, pp. 88-91, 1975.

[3] K. S. K. Iyengar, "A tauberian theorem and its application to convergence of Fourier series," Proceedings of the Indian Academy of Sciences: Section A, vol. 18, no. 2, pp. 81-87, 1943.

[4] J. A. Siddiqi, "On the harmonic summability of Fourier series," Proceedings of the Indian Academy of Sciences: Section A, vol. 28, no. 6, pp. 527-531, 1948.
[5] T. A. Pati, "Generalization of a theorem of Iyengar on the harmonic summability of Fourier series," Indian Journal of Mathematics, vol. 3, pp. 85-90, 1961.

[6] S. Lal and J. K. Kushwaha, "Degree of approximation of lipschitz function by product summability method," International Mathematical Forum, vol. 41, pp. 2101-2107, 2009.

[7] C. T. Rajagopal, "On the nörlund summability of Fourier series," Mathematical Proceedings of the Cambridge Philosophical Society, vol. 59, pp. 47-53, 1963.

[8] G. Alexits, "Über die Annäherung einer stetigen Funktion durch die Cesàroschen Mittelihrer Fourierreihe," Mathematische Annalen, vol. 100, no. 1, pp. 264-277, 1928.

[9] A. S. B. Hölland, B. N. Sahney, and J. Tzimbalario, "On the degree of approximation of a class of functions by means of Fourier series," Acta Scientiarum Mathematicarum, vol. 38, pp. 69-72, 1976. 


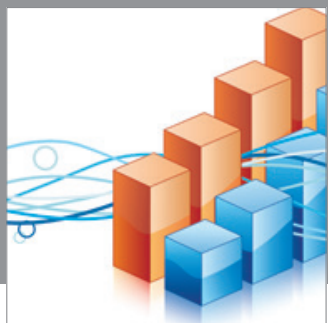

Advances in

Operations Research

mansans

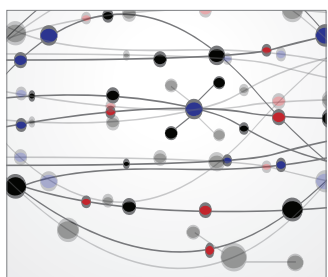

The Scientific World Journal
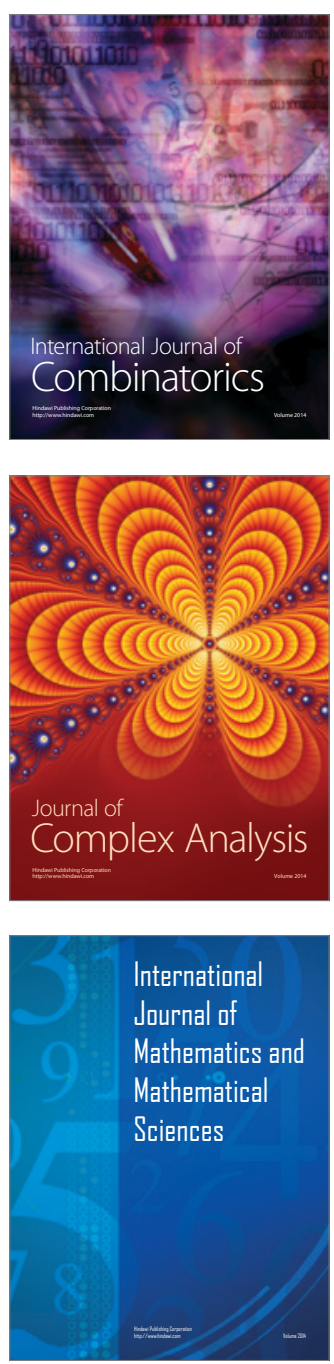
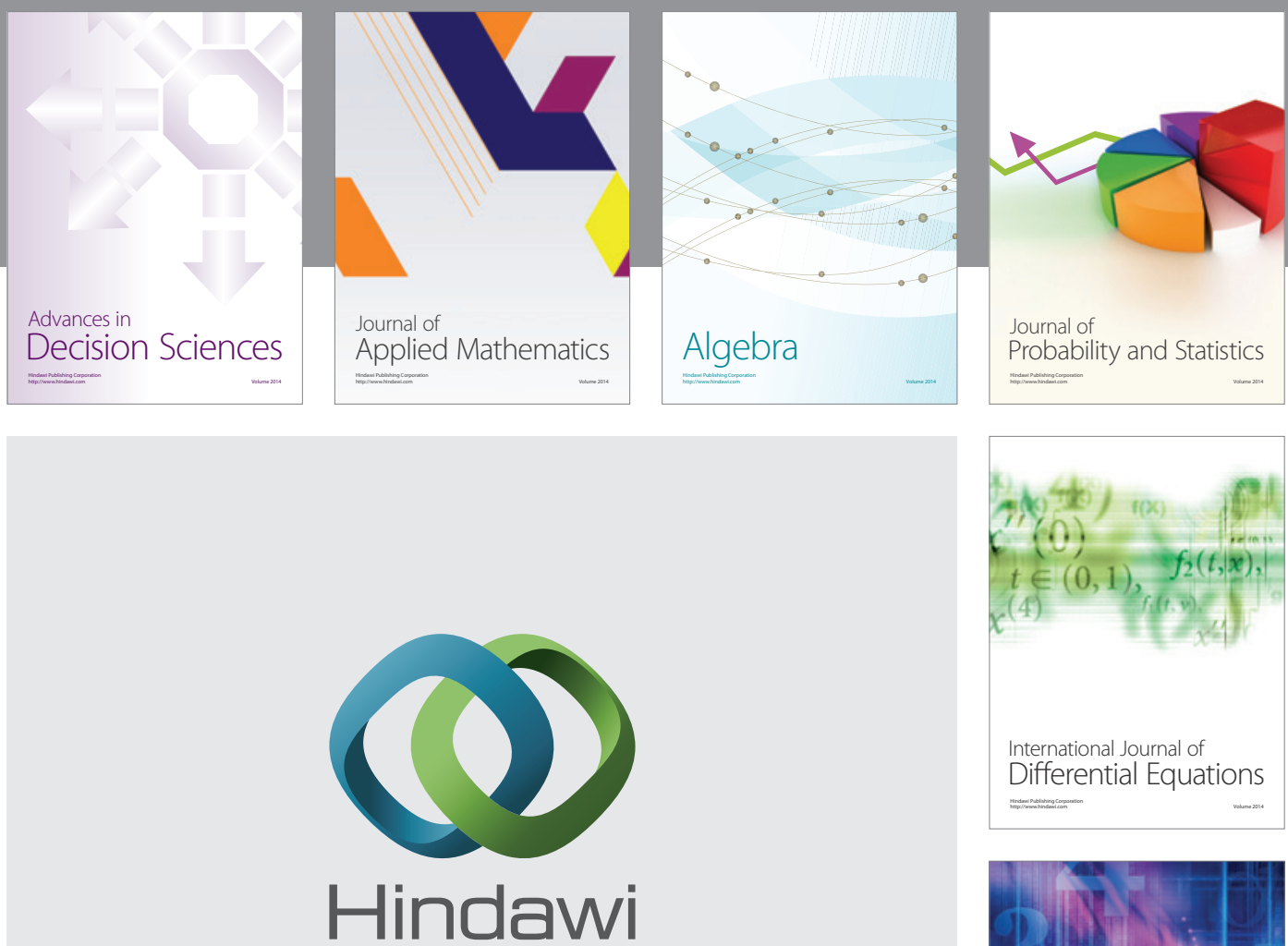

Submit your manuscripts at http://www.hindawi.com
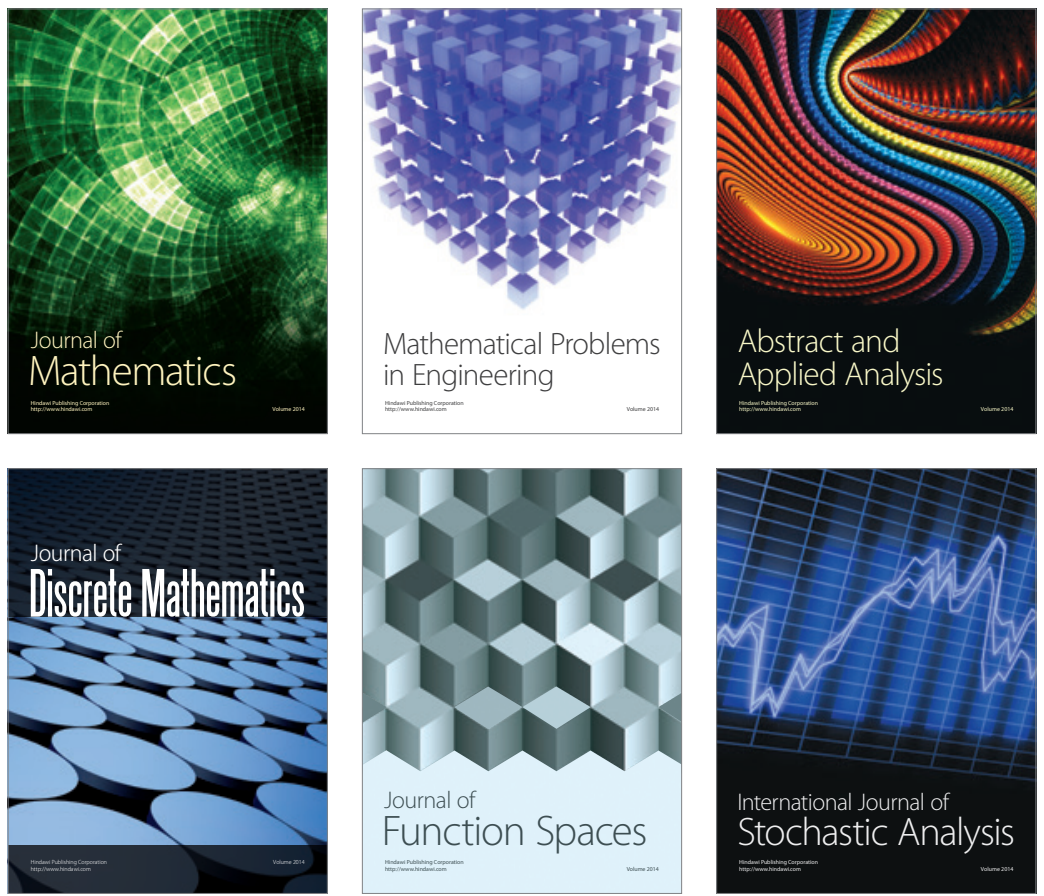

Journal of

Function Spaces

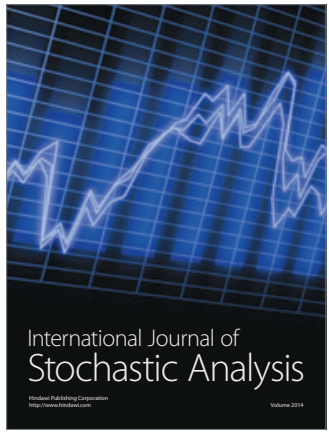

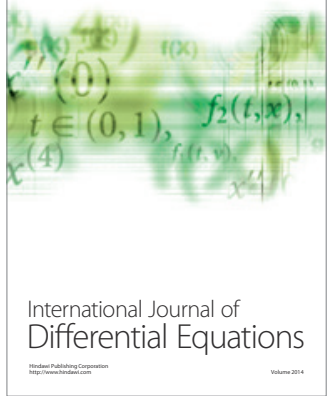
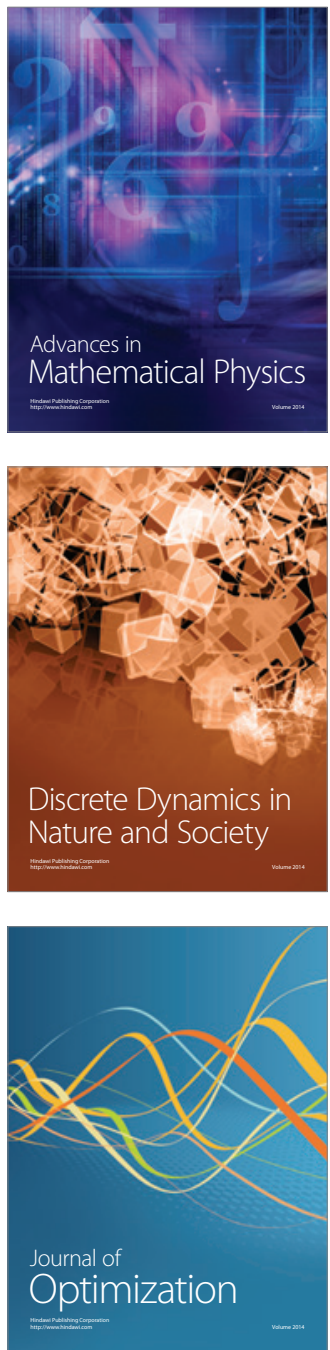